\title{
Blood Urea Nitrogen to Serum Albumin Ratio in the Prediction of Acute Kidney Injury of Patients with Rib Fracture in Intensive Care Unit
}

\author{
Tao He ${ }^{1, *}$, Gang $\mathrm{Li}^{2, *}$, Shoujia Xu', Leyun Guo', Bing Tang' \\ 'Department of Orthopedics, Sinopharm Dongfeng General Hospital, Hubei University of Medicine, Shiyan, Hubei, 442008, People's Republic of \\ China; ${ }^{2}$ Department of Sports Medicine, Sinopharm Dongfeng General Hospital, Hubei University of Medicine, Shiyan, Hubei, 442008, People's \\ Republic of China
}

*These authors contributed equally to this work

Correspondence: Leyun Guo; Bing Tang, Department of Orthopedics, Sinopharm Dongfeng General Hospital, Hubei University of Medicine, Daling Road 16\#, Shiyan, Hubei, 442008, People's Republic of China, Tel +86 0719-8210666, Email guoleyun@sohu.com; tangbingsy@I63.com

\begin{abstract}
Background: We hypothesized that the blood urea nitrogen (BUN) to serum albumin ratio (BAR) could serve as an independent predictor for incident acute kidney injury (AKI) in intensive care unit (ICU) patients with rib fracture.

Methods: Rib fracture patients in ICU were extracted from Medical Information Mart for Intensive Care IV (MIMIC-IV v1.0) database. The primary outcome in this study was the incidence of AKI. Univariate and multivariate logistic regression analyses were used to determine the relationship between BAR and AKI and propensity score matching (PSM) and inverse probability of treatment weighting (IPTW) were also applied to assure the robustness of our results.

Results: The optimal cut-off value for BAR was 5.26 based on receiver operator characteristic curve. Among the 953 patients who diagnosed with rib fracture, 197 high-BAR group ( $\geq 5.26)$ patients and 197 low-BAR group $(<5.26)$ patients who had similar propensity scores were finally included in the matched cohort. High-BAR group patients had a significantly higher incidence of AKI (odds ratio, OR, 3.85, 95\% confidence index, 95\% CI, 2.58-5.79, $\mathrm{P}<0.001)$ in the original cohort, in the matched cohort $(\mathrm{OR}$, 4.47, 95\% CI 2.71-7.53, P<0.001), and in the weighted cohort (OR, 4.28, 95\% CI 2.80-6.53, P<0.001). Furthermore, BAR was superior to that of acute physiology score III for predicting AKI and could add more net benefit for incident AKI in critical care patients with rib fracture.
\end{abstract}

Conclusion: As an easily access and cost-effective parameter, BAR could serve as a good diagnostic predictor for AKI in ICU patients with rib fracture.

Keywords: blood urea nitrogen to serum albumin ratio, rib fracture, intensive care unit, acute kidney injury, medical information mart for intensive care

\section{Introduction}

Acute kidney injury (AKI) is one of the most common disorders in intensive care units (ICU) worldwide and has been demonstrated to be a frustrating disease with high morbidity, mortality, healthcare cost and limited treatment options. ${ }^{1-4}$ Increasing evidences advocate that identifying patients with AKI at an early phase may improve the clinical outcomes of AKI patients after appropriate interventions be conducted. ${ }^{5,6}$ Therefore, a biomarker with highly accuracy and easily accessible may be crucial for clinicians to immediately and appropriately decision-making. Compared with the common reason for AKI, sepsis and cardiac surgery, for example, patients with fracture may be the rare one. ${ }^{7,8}$ However, more and more studies paid their attention to the development of AKI in hip or femoral neck fracture patients following surgery, ${ }^{9-13}$ and limited study had been published before to investigate the incident AKI in rib fracture patients in ICU.

Serum albumin is an easily accessible index for nutritional status and has been found to be associated with the prognosis of fractured patients. ${ }^{14-16}$ Blood urea nitrogen (BUN) is a simple but important index, combination with serum 
creatinine, to reflect kidney function and has also been found to be a prognostic factor for mortality in various diseases. ${ }^{17,18}$ Moreover, the BUN to serum albumin ratio (BAR), which is combined BUN and serum albumin, had introduced to be an important predictor for mortality in different types of disease. ${ }^{19,20}$ Nevertheless, as far as we can see, no study published before was made to evaluate the relationship between BAR and incident AKI for rib fracture patients. Therefore, in this study, we firstly demonstrated the relationship between BAR and the development of AKI for rib fracture patients from a freely public database, the Medical Information Mart for Intensive Care IV (MIMIC-IV version 1.0) database. Moreover, propensity score matching (PSM) and propensity score-based inverse probability of treatment weighting (IPTW) were also applied to assure the robustness of our findings.

\section{Materials and Methods}

\section{Study Population}

All data in this study were extracted from the MIMIC IV database, ${ }^{21}$ which consists of the information of more than 70,000 patients in Beth Israel Deaconess Medical Center. After successfully accomplishing the National Institutes of Health's (NIH) online training course and the Protection of Human Research Participants Examination, we had the access to extract data from MIMIC IV database. What is more, the study protocol was approved by the Ethics Committee of Sinopharm Dongfeng General Hospital and was waived to informed consent.

\section{Selection of Participants}

Adult patients ( $\geq 18$ years old) who were diagnosed as rib fracture based on the International Classification of Diseases (ICD) code were initially enrolled in this retrospective study. We only included patients who admitted to hospital and ICU firstly and we also excluded patients whose hospital stay less than 48 hours. Moreover, patients with end-stage renal disease (ESRD) or with missing BUN or serum albumin values were also excluded in this study. Finally, there were 953 patients diagnosed with rib fracture were included in the current study (Figure 1).

\section{Variable Extraction}

Demographic and admission information: age, gender, body mass index (BMI), number of rib fracture, and severity scores and the Charlson comorbidity index were also calculated. Commodities including hypertension, diabetes, chronic kidney disease (CKD), congestive heart failure (CHF), myocardial infarct, chronic obstructive pulmonary disease (COPD), liver disease and malignancy were also extracted based on the ICD codes. Use of mechanical ventilation (MV), vasopressors and renal replacement therapy during their first day of hospital stay were also recorded in this study. Moreover, initial vital signs and laboratory results were also extracted by structured query language with PostgreSQL 9.6.

The BAR was calculated by initial serum BUN $(\mathrm{mg} / \mathrm{dL}) /$ serum albumin $(\mathrm{g} / \mathrm{dL})$.

The primary outcome in this study was the incident of AKI in 48 hours, which was stratified based on its severity, according to the KDIGO Clinical Practice Guidelines for AKI. ${ }^{22}$

\section{Statistical Analyses}

Continuous variables were expressed as mean (standardized mean difference, SMD), categorical covariates were reported as number (percentage). Receiver operator characteristic curve (ROC) and Youden index were used to identify the best cutoff values of BAR for AKI. PSM and IPTW were also used to adjust the imbalance of the covariates between two groups. Multivariate logistic regression and adjusted odds ratio (OR) were calculated in this study to determine the relationship between BAR and the incident AKI. Discrimination was also assessed by the integrated discrimination index (IDI). Improvement in clinical risk stratification was assessed by calculating net reclassification improvement (NRI). Moreover, the decision curve analysis (DCA) was performed to assess the clinical benefits of the BAR. All analyses were conducted using $\mathrm{R}$ (version 4.1.0) and $\mathrm{p}<0.05$ was considered statistically significant. 


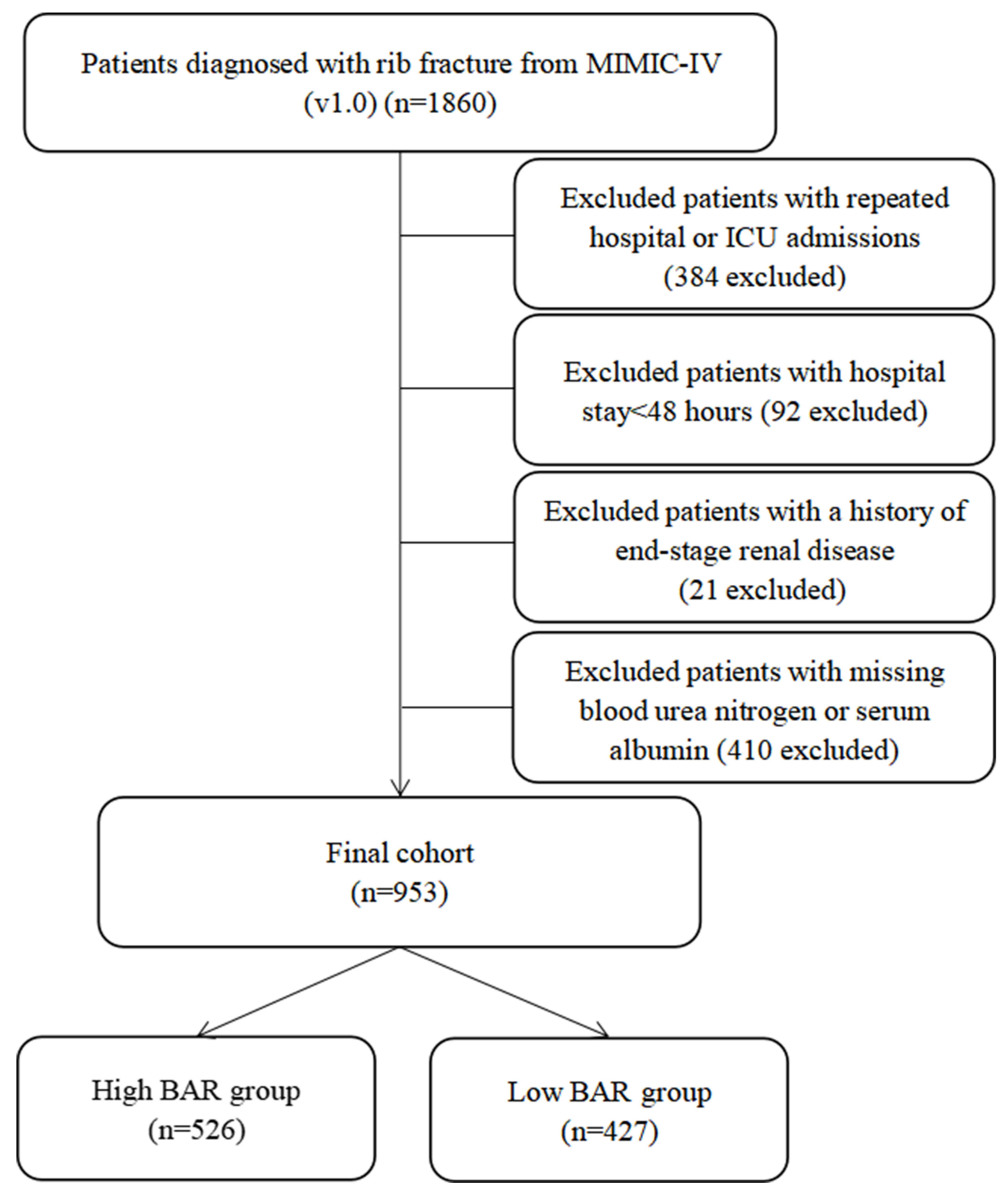

Figure I Study flow diagram in the present study.

\section{Results}

\section{Patient Clinical Features}

Finally, a total of 953 patients with rib fracture were included in this study (Figure 1). Among them, AKI occurred in 459 (48.2\%) patients. According to the KDIGO criteria, 104 (10.9\%) patients were AKI stage I, 166 (17.4\%) patients were AKI stage II and 89 (9.3\%) patients were AKI stage III.

Based on the optimal cut-off value of BAR (5.26), which was determined by ROC analysis, we grouped patients as high BAR group $(\geq 5.26, \mathrm{n}=427)$ and low BAR group $(<5.26, \mathrm{n}=526)$. The baseline characteristics of the high BAR group and low BAR group are summarized in Table 1. In original cohort, 25/35 covariates (age, use of MV, CRRT and vasopressors, OASIS score, APSIII score, SOFA score, SAPSII score, Charlson score, hypertension, diabetes, CKD, myocardial infarct, CHF, liver disease, white blood cells, hemoglobin, platelet, total bilirubin, anion gap, creatinine, glucose, potassium, mean arterial pressure, and $\mathrm{SpO}_{2}$ ) were imbalanced between high BAR group and low BAR group. Based on the estimated propensity scores, PSM and IPTW were used to standardize the differences between the two groups. Moreover, the imbalance between the two groups was significantly decreased and all variables were comparable between high BAR group and low BAR group (Table 1 and Supplemental Figure 1). 
Table I Comparisons of Baseline Characteristics Between the Original Cohort, Matched Cohort and Weighted Cohort

\begin{tabular}{|c|c|c|c|c|c|c|c|c|c|}
\hline \multirow[t]{2}{*}{ Covariates } & \multicolumn{3}{|c|}{ Original Cohort } & \multicolumn{3}{|c|}{ Matched Cohort } & \multicolumn{3}{|c|}{ Weighted Cohort } \\
\hline & Low BAR & High BAR & $\mathbf{P}$ & Low BAR & High BAR & $\mathbf{P}$ & Low BAR & High BAR & $\mathbf{P}$ \\
\hline $\mathrm{N}$ & 526 & 427 & & 197 & 197 & & 189.8 & 192.9 & \\
\hline Age, years & $53.5(19.5)$ & $70.6(16.6)$ & $<0.001$ & $66.3(16.5)$ & $64.7(17.5)$ & 0.353 & $64.9(17.3)$ & $64.5(17.7)$ & 0.782 \\
\hline Gender, male, n (\%) & $334(63.5)$ & $266(62.3)$ & 0.753 & 116 (58.9) & $122(61.9)$ & 0.607 & $117.5(61.9)$ & $120.1(62.3)$ & 0.934 \\
\hline $\mathrm{BMI}, \mathrm{kg} / \mathrm{m}^{2}$ & $26.8(6.3)$ & $27.1(7.5)$ & 0.483 & $26.5(6.1)$ & $27.1(7.3)$ & 0.409 & $26.9(6.3)$ & $26.9(7.1)$ & 0.969 \\
\hline Number of fracture, (\%) & & & 0.171 & & & 0.972 & & & 0.753 \\
\hline I & $106(20.2)$ & $70(16.4)$ & & $35(17.8)$ & $33(16.8)$ & & $33.6(17.7)$ & $28.5(14.8)$ & \\
\hline $2-3$ & $78(14.8)$ & $57(13.3)$ & & $26(13.2)$ & $24(12.2)$ & & $25.8(13.6)$ & $27.5(14.3)$ & \\
\hline 4 or more & $140(26.6)$ & $107(25.1)$ & & $51(25.9)$ & $51(25.9)$ & & $53.0(27.9)$ & $51.2(26.5)$ & \\
\hline Multiple & $202(38.4)$ & $193(45.2)$ & & $85(43.1)$ & $89(45.2)$ & & $77.4(40.8)$ & $85.7(44.4)$ & \\
\hline \multicolumn{10}{|l|}{ Interventions, $\mathrm{n}(\%)$} \\
\hline MV & $221(42.0)$ & $235(55.0)$ & $<0.001$ & 95 (48.2) & $95(48.2)$ & 1.000 & 93.1 (49.0) & 93.9 (48.7) & 0.934 \\
\hline CRRT & $3(0.6)$ & $23(5.4)$ & $<0.001$ & $2(1.0)$ & I (0.5) & 1.000 & $2.2(1.1)$ & $1.8(0.9)$ & 0.804 \\
\hline Vasopressors & $99(18.8)$ & 191 (44.7) & $<0.001$ & $67(34.0)$ & $67(34.0)$ & 1.000 & $61.3(32.3)$ & $62.3(32.3)$ & 0.999 \\
\hline \multicolumn{10}{|l|}{ Severity score, points } \\
\hline SOFA & $3.6(1.0)$ & $6.2(2.1)$ & $<0.001$ & $4.7(1.3)$ & $4.8(1.6)$ & 0.739 & $4.7(2.3)$ & $4.7(2.5)$ & 0.989 \\
\hline OASIS & $29.9(8.0)$ & $35.7(9.0)$ & $<0.001$ & $33.6(7.6)$ & $33.1(8.0)$ & 0.558 & $33.3(7.9)$ & $33.1(8.0)$ & 0.792 \\
\hline APSIII & $38.1(18.7)$ & $55.8(24.1)$ & $<0.001$ & $47.1(21.9)$ & $47.2(19.8)$ & 0.948 & $47.0(22.1)$ & $46.9(19.5)$ & 0.973 \\
\hline SAPSII & $25.8(I I .2)$ & $40.1(13.6)$ & $<0.001$ & $34.1(10.0)$ & 33.7 (II.8) & 0.690 & $33.3(10.6)$ & $33.1(10.6)$ & 0.809 \\
\hline \multicolumn{10}{|l|}{ Comorbidities, n (\%) } \\
\hline Hypertension & $176(33.5)$ & $174(40.7)$ & 0.024 & $87(44.2)$ & $88(44.7)$ & 1.000 & $84.5(44.5)$ & $86.0(44.6)$ & 0.995 \\
\hline Diabetes & $65(12.4)$ & $93(21.8)$ & $<0.001$ & $34(17.3)$ & $39(19.8)$ & 0.604 & $32.1(16.9)$ & $32.4(16.8)$ & 0.975 \\
\hline CKD & $8(1.5)$ & $63(14.8)$ & $<0.001$ & $6(2.0)$ & II (5.6) & 0.321 & $5.9(3.1)$ & $7.4(3.9)$ & 0.647 \\
\hline Myocardial infarct & $20(3.8)$ & $58(13.6)$ & $<0.001$ & $16(8.1)$ & $17(8.6)$ & 1.000 & $15.5(8.2)$ & $15.8(8.2)$ & 0.997 \\
\hline $\mathrm{CHF}$ & $31(5.9)$ & $109(25.5)$ & $<0.001$ & $26(13.2)$ & $26(13.2)$ & 1.000 & $24.9(13.1)$ & $24.5(12.7)$ & 0.883 \\
\hline COPD & $93(17.7)$ & $74(17.3)$ & 0.955 & $34(17.3)$ & $38(19.3)$ & 0.696 & $34.3(18.0)$ & $35.7(18.5)$ & 0.899 \\
\hline Liver disease & $34(6.5)$ & $56(13.1)$ & 0.001 & $20(10.2)$ & $18(9.1)$ & 0.864 & $18.8(9.9)$ & $18.8(9.7)$ & 0.951 \\
\hline Malignancy & $22(4.2)$ & $20(4.7)$ & 0.829 & $7(3.6)$ & $9(4.6)$ & 0.799 & $8.2(4.3)$ & $8.2(4.3)$ & 0.988 \\
\hline Charlson index & $2.7(1.3)$ & $5.2(2.7)$ & $<0.001$ & $4.2(1.3)$ & $4.1(2.4)$ & 0.761 & $4.1(1.3)$ & $4.0(1.3)$ & 0.962 \\
\hline \multicolumn{10}{|l|}{ Vital signs } \\
\hline MAP, mmHg & $90.7(17.0)$ & $86.1(20.2)$ & $<0.001$ & $88.7(17.0)$ & $88.9(21.8)$ & 0.910 & $89.2(17.4)$ & $88.9(19.2)$ & 0.835 \\
\hline Heart rate, bpm & 91.7 (18.6) & 89.4 (19.8) & 0.070 & $89.7(18.5)$ & $89.3(18.5)$ & 0.834 & $89.6(18.6)$ & $89.3(19.2)$ & 0.835 \\
\hline $\mathrm{RR}, \mathrm{bpm}$ & $19.6(5.8)$ & $20.0(5.5)$ & 0.346 & $20.4(6.8)$ & $19.9(5.1)$ & 0.400 & $20.1(6.3)$ & $20.0(5.2)$ & 0.794 \\
\hline $\mathrm{SpO} 2, \%$ & $97.3(3.4)$ & $96.5(4.3)$ & 0.001 & $96.8(4.1)$ & $96.7(4.3)$ & 0.783 & $96.9(4.1)$ & 96.9 (3.9) & 0.963 \\
\hline \multicolumn{10}{|l|}{ Laboratory results } \\
\hline WBC, $\times 10^{9} / \mathrm{L}$ & $12.2(5.3)$ & I3.4 (5.6) & 0.019 & $12.2(5.6)$ & $12.3(5.4)$ & 0.865 & $12.4(5.7)$ & $12.5(5.8)$ & 0.986 \\
\hline $\mathrm{HGB}, \mathrm{g} / \mathrm{dL}$ & $11.8(2.0)$ & $10.9(2.1)$ & $<0.001$ & II.3 (2.0) & $11.3(2.0)$ & 0.769 & II.4 (2.0) & $11.3(2.1)$ & 0.856 \\
\hline $\mathrm{PLT}, \times 10^{9} / \mathrm{L}$ & $202.9(77.3)$ & $190.0(66.1)$ & 0.015 & $193.2(78.3)$ & $194.8(78.8)$ & 0.837 & $195.2(78.2)$ & $194.9(83.0)$ & 0.966 \\
\hline Glucose, mg/dl & I36.3 (49.6) & $162.0(76.2)$ & $<0.001$ & I $48.8(65.1)$ & $151.4(62.9)$ & 0.684 & $150.4(64.9)$ & $151.4(63.6)$ & 0.863 \\
\hline Bilirubin, mmol/L & I.I (0.5) & $\mathrm{I} .4(0.7)$ & 0.032 & $1.2(0.4)$ & $1.3(0.3)$ & 0.687 & $1.2(0.4)$ & $1.2(0.3)$ & 0.855 \\
\hline Anion gap, mEq/L & $14.7(3.6)$ & $15.2(4.1)$ & 0.030 & $14.8(3.7)$ & 14.7 (3.6) & 0.890 & I4.7 (3.7) & I 4.7 (3.6) & $0.88 I$ \\
\hline Bicarbonate, $\mathrm{mEq} / \mathrm{L}$ & $22.9(3.9)$ & $22.5(4.2)$ & 0.192 & $22.8(4.3)$ & $22.7(3.8)$ & 0.802 & $22.9(4.2)$ & $22.8(4.0)$ & 0.942 \\
\hline Chloride, $\mathrm{mmol} / \mathrm{L}$ & $104.2(6.0)$ & $104.6(6.4)$ & 0.421 & $104.6(6.5)$ & $104.5(6.1)$ & 0.861 & $104.6(6.3)$ & $104.6(6.2)$ & 0.929 \\
\hline Creatinine, mg/dL & $0.8(0.3)$ & $1.3(0.7)$ & $<0.001$ & $0.9(0.4)$ & $1.0(0.4)$ & 0.069 & $1.0(0.4)$ & $1.0(0.3)$ & 0.636 \\
\hline Potassium, mmol/L & $4.1(0.7)$ & $4.4(0.8)$ & $<0.001$ & $4.3(0.8)$ & $4.3(0.8)$ & 0.652 & $4.3(0.8)$ & $4.3(0.7)$ & 0.905 \\
\hline Sodium, mmol/L & $138.6(4.6)$ & $139.0(5.0)$ & 0.229 & $138.9(5.0)$ & I 38.7 (4.5) & 0.656 & I 38.8 (4.9) & I38.9 (4.6) & 0.996 \\
\hline \multicolumn{10}{|l|}{ Clinical outcomes } \\
\hline AKI stages, n (\%) & & & $<0.001$ & & & $<0.001$ & & & $<0.001$ \\
\hline No AKI & $370(80.3)$ & $124(29.0)$ & & $12 \mid(6 \mid .4)$ & $71(36.0)$ & & II $9.4(62.9)$ & $70.9(36.7)$ & \\
\hline
\end{tabular}

(Continued) 
Table I (Continued).

\begin{tabular}{|c|c|c|c|c|c|c|c|c|c|}
\hline \multirow[t]{2}{*}{ Covariates } & \multicolumn{3}{|c|}{ Original Cohort } & \multicolumn{3}{|c|}{ Matched Cohort } & \multicolumn{3}{|c|}{ Weighted Cohort } \\
\hline & Low BAR & High BAR & $\mathbf{P}$ & Low BAR & High BAR & $\mathbf{P}$ & Low BAR & High BAR & $\mathbf{P}$ \\
\hline Stage I & $40(7.6)$ & $64(15.0)$ & & $22(11.2)$ & $31(15.7)$ & & $19.0(10.0)$ & $32.7(17.0)$ & \\
\hline Stage II & $93(17.7)$ & $173(40.5)$ & & $4 \mathrm{I}(20.8)$ & $81(4 I .1)$ & & $39.1(20.6)$ & $76.7(39.8)$ & \\
\hline Stage III & $23(4.4)$ & $66(I 5.5)$ & & $13(6.6)$ & $14(7.1)$ & & $12.2(6.4)$ & $12.6(6.5)$ & \\
\hline
\end{tabular}

Notes: For all continuous covariates, the mean values and standard deviations are reported.

Abbreviations: BAR, blood urea nitrogen to serum albumin ratio, BMI, body mass index, MV, mechanical ventilation, CRRT, continuous renal replacement therapy, SOFA, sequential organ failure assessment, OASIS, Oxford Acute Severity of IIIness Score, APSIII, Acute Physiology Score III, SAPSII, Simplified Acute Physiology Score II, CKD, chronic kidney disease, CHF, congestive heart failure, COPD, chronic obstructive pulmonary disease, MAP, mean arterial pressure, RR, respiratory rate, WBC, white blood cell, HGB, hemoglobin, PLT, platelet, AKI, acute kidney injury.

Table 2 Univariate and Multivariate Logistic Analysis of BAR for the Development of AKI

\begin{tabular}{|c|c|c|c|c|c|c|}
\hline & \multicolumn{2}{|l|}{ Original Cohort } & \multicolumn{2}{|c|}{ Matched Cohort } & \multicolumn{2}{|c|}{ Weighted Cohort } \\
\hline & OR $(95 \% \mathrm{Cl})$ & $P$ value & OR (95\% Cl) & $P$ value & OR $(95 \% \mathrm{CI})$ & $P$ value \\
\hline Unadjusted & $5.80(4.39-7.69)$ & $<0.001$ & $3.0 \mathrm{I}(2.0 \mathrm{I}-4.56)$ & $<0.001$ & $2.92(2.03-4.21)$ & $<0.001$ \\
\hline Model I & $5.98(4.34-8.32)$ & $<0.001$ & $3.18(2.08-4.92)$ & $<0.001$ & $3.18(2.17-4.65)$ & $<0.001$ \\
\hline Model 2 & $5.45(3.91-7.65)$ & $<0.001$ & $3.22(2.09-5.02)$ & $<0.001$ & $3.26(2.22-4.78)$ & $<0.001$ \\
\hline Model 3 & $3.88(2.66-5.7 I)$ & $<0.001$ & $4.20(2.59-6.95)$ & $<0.001$ & $4.07(2.7 I-6.10)$ & $<0.001$ \\
\hline Model 4 & $3.85(2.58-5.79)$ & $<0.001$ & $4.47(2.7 I-7.53)$ & $<0.001$ & $4.28(2.80-6.53)$ & $<0.001$ \\
\hline
\end{tabular}

Notes: Model I adjusted for age, gender, body mass index and number of fracture. Model 2 adjusted for model I plus comorbidities. Model 3 adjusted for Model 2 plus score systerm, interventions and Charlson index. Model 4 adjusted for model 3 plus vital signs and laboratory results.

Abbreviations: $\mathrm{AKI}$, acute kidney injury, OR, odds ratio, $95 \% \mathrm{CI}, 95 \%$ confidence index.

\section{BAR as a Predictor for the Primary End Point}

Compared patients in low-BAR group, high-BAR group patients had a relatively higher incidence of AKI in original set (71.0\% vs $19.7 \%, \mathrm{P}<0.001)$, in matched set $(64.0 \%$ vs $38.6 \%, \mathrm{P}<0.001)$ and in weighted set $(63.3 \%$ vs $37.1 \%, \mathrm{P}<0.001)$. Moreover, the Spearman analysis also indicates that BAR was positively correlated with AKI stage in the original cohort $(r=0.411, \mathrm{P}<0.001)$ and in the matched cohort $(r=0.324, \mathrm{P}<0.001)$.

Univariate logistic analysis showed that patients in high BAR group had increased incident of AKI, with the crude odds ratio (OR) was 5.80 (95\% confidence index, 95\% CI, 4.39-7.69, $\mathrm{P}<0.001)$ and the relationship remained robust after PSM (OR, 3.01, 95\% CI 2.01-4.56, P<0.001) and IPTW (OR, 2.92, 95\% CI 2.03-4.21, P<0.001) (Table 2). This association was further verified in multivariate analyses. BAR could still serve as a significant predictor for incident AKI in original cohort (OR, 3.85, 95\% CI 2.58-5.79, $\mathrm{P}<0.001$ ), in matched cohort (OR, 4.47, $95 \%$ CI 2.71-7.53, $\mathrm{P}<0.001)$ as well as in weighted cohort (OR, 4.28, 95\% CI 2.80-6.53, P<0.001) after adjustment for confounding features (Table 2). Those results demonstrated that BAR was an independent and robust predictor for AKI in rib fracture patients.

To further confirm whether BAR remained an independent factor for AKI, we also performed subgroup analyses. Forest plot demonstrated that high BAR was associated with high incidence of AKI in most subgroups (Figure 2A). Furthermore, the results in matched cohort also verify our results (Figure 2B).

\section{Effect of BAR on Risk Reclassification of AKI}

NRI and IDI were introduced to verify the hypothesis that BAR could improve the risk reclassification of incident AKI in critical care patients with rib fracture. And not surprisingly, compared with APSIII score, BAR could significantly improve the risk reclassification for incident of AKI in the original set as well as in the matched set (Table 3). 

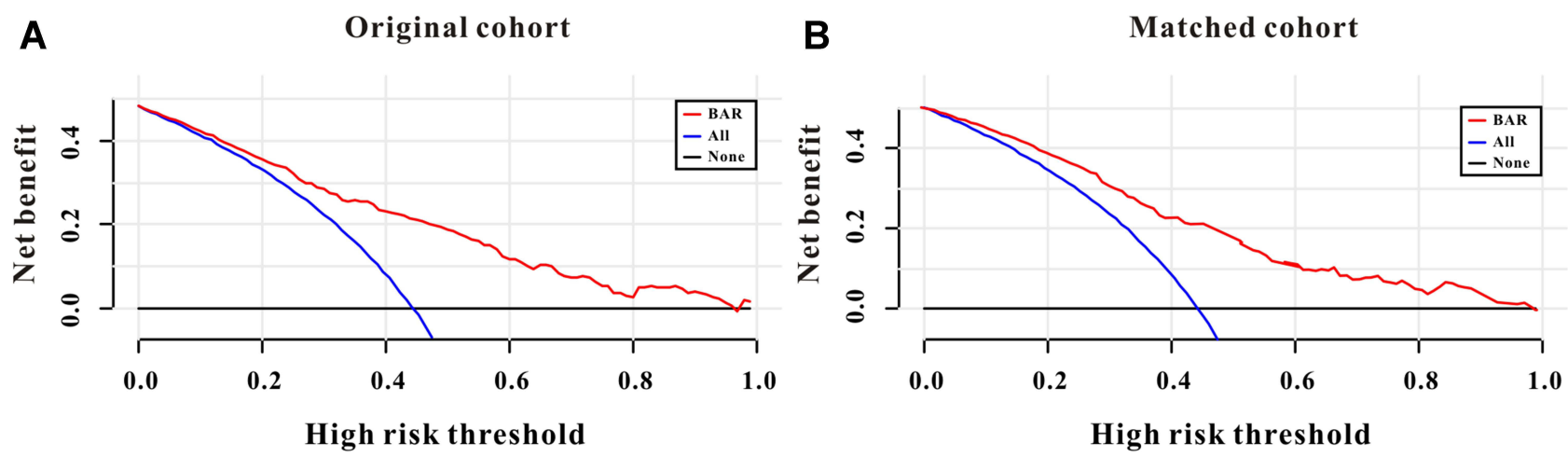

Figure 2 Decision curve analysis of BAR for incident AKI in rib fracture patients to detect its clinical usefulness in the original cohort (A) and in the matched cohort (B).

\section{Clinical Usefulness of BAR}

DCA curve was also applied to assess the clinical usefulness of BAR for incident of AKI. As described in Figure 3, BAR could add more net benefit than the "treat all" or "treat none" strategies both in the original set and in the matched set (Figure 3A and B). Therefore, these results demonstrated that BAR was clinical usefulness.

\section{Discussion}

In this retrospective study of 953 rib fracture patients from a freely public database and found that patients with high BAR had increased incidence of AKI and demonstrated that initial BAR could serve as a good predictor for incident AKI even after PSM and IPTW were applied to adjust the imbalance of the covariates. Moreover, BAR was superior to that of APSIII score for predicting AKI and could added more net benefit for AKI than treat all or treat none strategies. Therefore, our findings concluded that the initial BAR might have good diagnostic ability for incident AKI in rib fracture patients in ICU.

The incidence of AKI in fracture patients had been described in previous studies, mostly were hip fracture following surgery. ${ }^{23-26}$ Rantalaiho et al performed a retrospectively multicenter study of 486 consecutive low-energy trauma hip fracture patients and found that a percent of $8.4 \%$ patients were developed AKI and AKI patients had threefold threemonth mortality after surgery compared with patients without AKI. ${ }^{27}$ Another retrospective study of 299 patients aged above 65 years who underwent surgery concluded that the incidence of AKI was $28.4 \%$ and AKI was associated with longer hospital stay and increased mortality. ${ }^{25}$ Moreover, a recent meta-analysis consisting of 11 studies with 16,421 surgical hip fracture patients found the pooled prevalence of AKI was 17\% (95\% CI 14-21\%). ${ }^{28}$ Compared with those studies, the incidence of AKI in the current study was much higher (48.2\%) and might at least partly explained by differences in patient populations and severity of disease. Furthermore, rib fracture patients especially in ICU occasionally require surgical treatment and commonly complain about chest pain, atelectasis, hypoventilation, hypoxemia, respiratory failure and subsequent cardiac complications. Therefore, those subsequent cardiopulmonary complications may be another significant reason why the incidence of AKI is really high.

To the best of our knowledge, we had applied BAR for incident AKI in rib fracture patients in ICU for the first time and observed significant positive correlations between them. More importantly, the initial BAR was superior to that of APSIII score alone for predicting AKI and could add more net benefit for AKI than treat all or treat none. We could explain this as follows: first of all, as a rejected material of protein metabolism manufactured in the liver and evacuated by the kidney, BUN unusually used as one of the most common indexes to assess the renal function in clinical practice and BUN can also be affected by several conditions, such as protein intake, gastrointestinal bleeding, dehydration, inflammation, and so on. ${ }^{29}$ Increased BUN level may result from the reduction of water volume in the whole body, leading to higher risk of AKI and while decreased BUN level may be caused by protein deficiency or hepatic failure, both of them can increase the risk of AKI. ${ }^{30,31}$ Secondly, ALB maintains the body's nutrition and osmotic pressure as one of the most crucial protein for mankind. Patients with low level of ALB are a sign of malnutrition and has been found to be associated with increased clinical outcomes in patients with different types of disease as well as AKI patients. ${ }^{32,33}$ Due to 
Table $3 \mathrm{NRI}$ and IDI Analyses for Risk Reclassification of AKI in the Original Cohort and in the Matched Cohort

\begin{tabular}{|c|c|c|c|c|c|c|c|c|c|c|}
\hline \multirow[t]{2}{*}{ Outcome } & \multirow[b]{2}{*}{ Sensibility (\%) } & \multirow[b]{2}{*}{ Specificity (\%) } & \multicolumn{4}{|c|}{ AUC } & \multicolumn{2}{|l|}{ IDI } & \multicolumn{2}{|l|}{$\mathbf{N R I ^ { \mathbf { a } }}$} \\
\hline & & & Biomarker & $\begin{array}{l}\text { Biomarker+ } \\
\text { Clinical Model }\end{array}$ & Clinical Model $^{\mathbf{b}}$ & P value ${ }^{c}$ & Value $(95 \% \mathrm{Cl})$ & $\begin{array}{c}P \\
\text { value }\end{array}$ & Value $(95 \% \mathrm{Cl})$ & $\begin{array}{c}P \\
\text { value }\end{array}$ \\
\hline \multicolumn{11}{|l|}{ Original cohort } \\
\hline BAR & 73.0 & 71.7 & 0.757 & 0.854 & 0.835 & $<0.001$ & $0.066(0.050-0.08 \mathrm{I})$ & $<0.001$ & $0.134(0.078-0.191)$ & $<0.001$ \\
\hline APSIII & 66.0 & 74.9 & 0.705 & 0.839 & & $<0.001$ & $0.009(0.003-0.016)$ & 0.045 & $0.034(-0.003-0.07$ I) & 0.075 \\
\hline$B A R+A P S I I I$ & 71.7 & 75.3 & 0.812 & 0.855 & & $<0.001$ & $0.075(0.058-0.092)$ & $<0.001$ & $0.172(0.111-0.233)$ & $<0.001$ \\
\hline \multicolumn{11}{|l|}{ Matched cohort } \\
\hline BAR & 71.3 & 60.2 & $0.7 I I$ & 0.837 & 0.789 & $<0.001$ & $0.105(0.07 \mid-0.132)$ & $<0.001$ & $0.172(0.07 I-0.270)$ & 0.0008 \\
\hline APSIII & 69.9 & 61.5 & 0.687 & 0.793 & & 0.0004 & $0.013(0.002-0.023)$ & 0.018 & $0.112(0.052-0.173)$ & 0.0003 \\
\hline BAR+APSIII & 85.4 & 52.3 & 0.767 & 0.841 & & $<0.001$ & $0.117(0.085-0.149)$ & $<0.001$ & $0.256(0.150-0.362)$ & $<0.001$ \\
\hline
\end{tabular}

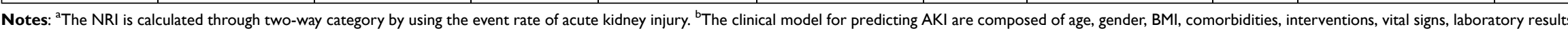
and score system except APSIII score. 'Biomarker+clinical model versus clinical model.

Abbreviations: AUC, area under the receiver-operating characteristic curve; IDI, integrated discrimination improvement; NRI, net reclassification index; BAR, blood urea nitrogen to serum albumin ratio, APSIII, Acute Physiology Score III. 
A

Characteristics
All case
Age
$\geqslant 60$ yr
<60 yr
Gender
Male
Female
Number of rib fracture
One
Two or three
Four or more
Multiple
Hypertension
Yes
No
Diabetes
Yes
No
CKD
Yes
No
COPD
Yes
No
CHF
Yes
No
Myocardial infarct
Yes
No
Liver disease
Yes
No
Malignancy
Yes
No

NoPo

B

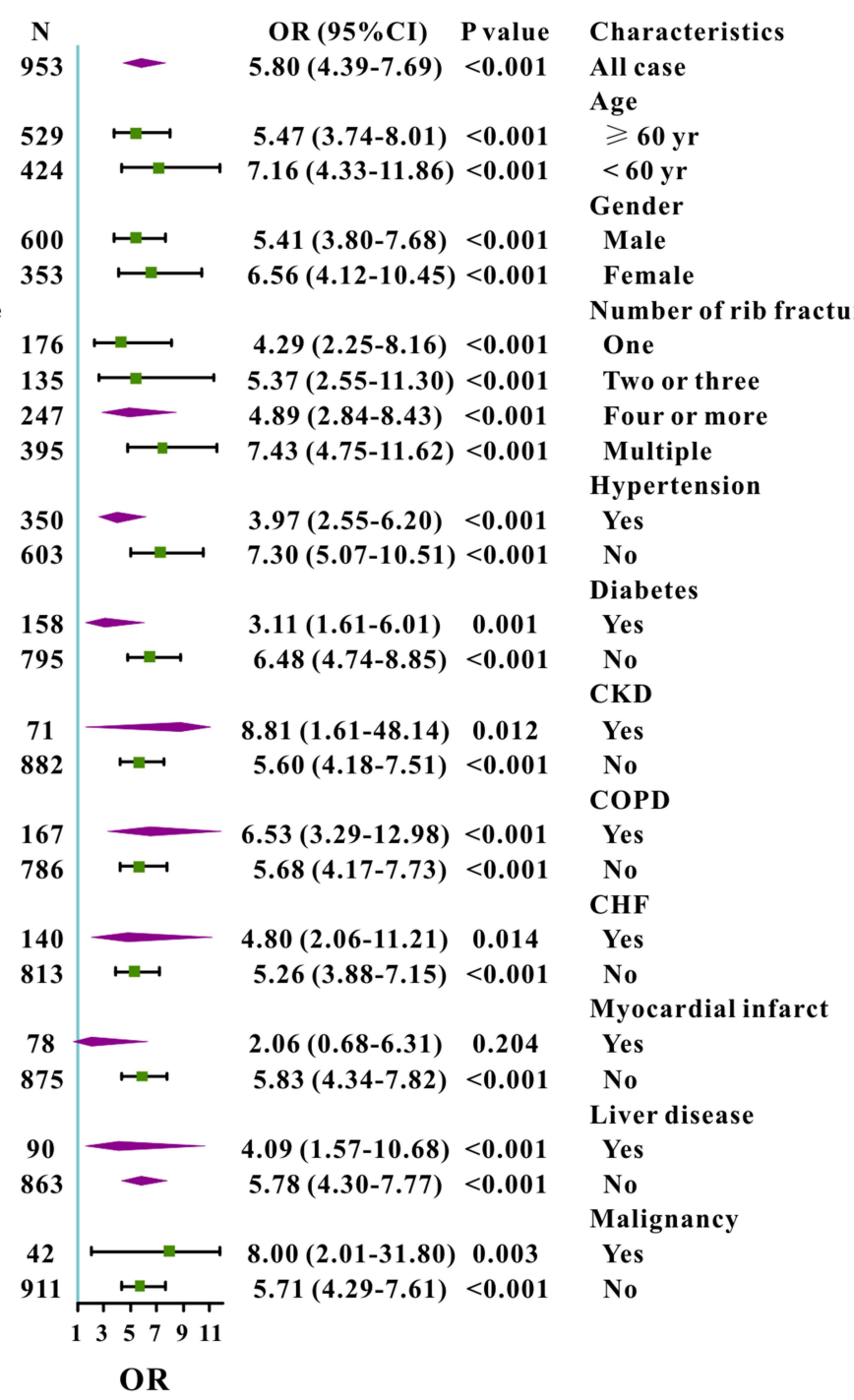

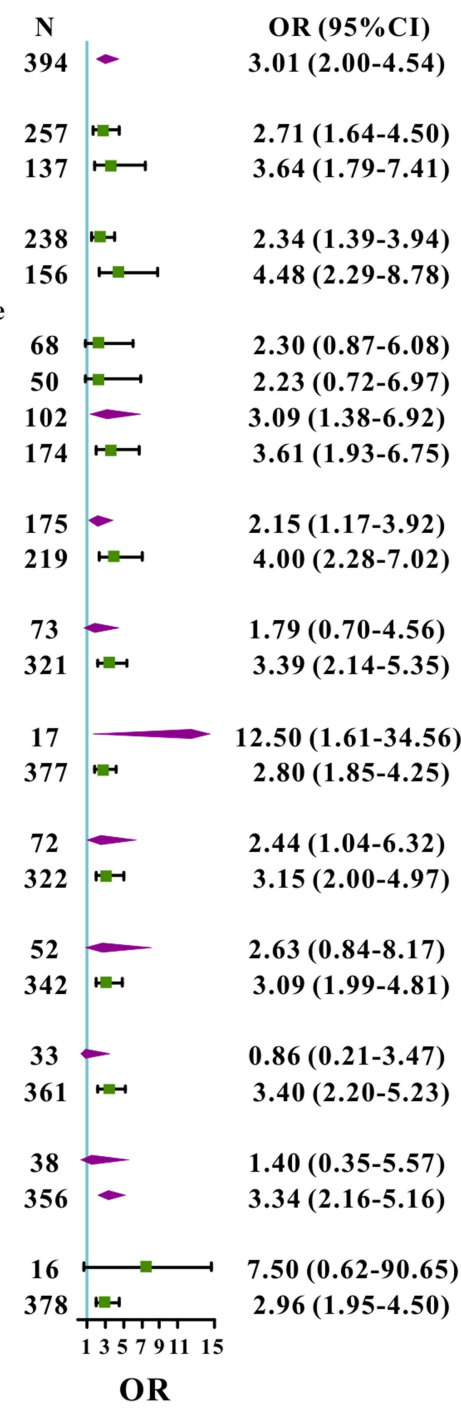

Figure 3 Subgroup analysis for odds ratio of rib fracture patients with BAR $\geq 5.26$ versus BAR $<5.26$ in different groups in the original cohort (A) and in the matched cohort (B).

the usage of some instruments or the limits of disease, patients in ICU usually show ALB reduction in serology, leading to the high risk of AKI. BUN and ALB are easily accessible and inexpensive and in the current study, we firstly combined BUN and ALB and found that initial BAR had superior diagnostic ability for AKI to that of APSIII score alone and could added more net benefit for AKI in DCA curve. Hence, BAR, an indicator of inflammation and nutritional status, may act as a new independent predictor for AKI.

However, some limitations also exist in this retrospective study. Firstly, only the initial BAR value was included in this study to access the diagnostic value for AKI while dynamic changes of it did not monitor in this study even those values may be more accurate for AKI. Secondly, due to the limit of the database, we did not obtain levels of serum C-reactive protein and some other inflammatory or nutritional indicators, which may be helpful to investigate the mechanism of the association between BAR and incident AKI for rib fracture patients in ICU. Moreover, serum BUN value might be affected by the diet structure; however, due to the database limit, we did not obtain the information about their diet structure. Finally, this was a retrospective study, and further perspective multicenter studies are proposed to validate the conclusions of our study and to investigate the potential mechanism behind them. 


\section{Conclusions}

In this retrospective study, based on a public database, we firstly concluded that initial BAR was a significant predictor for incident AKI in critical care patients with rib fracture, which was good discrimination and clinical usefulness. Considering that BAR was an easily access and cost-effective parameter, it might provide a helpful method for clinicians to stratify the risk of AKI. Despite the solid statistics of this retrospective study, further studies have been proposed to validate the results of our study.

\section{Data Sharing Statement}

All data in our study are available from the corresponding author upon reasonable request.

\section{Statement of Ethics}

This study was conducted in accordance with the Helsinki Declaration, and authors had successfully accomplished the National Institutes of Health's (NIH) online training course and the Protection of Human Research Participants Examination and got the permission to extract data from MIMIC IV database. Moreover, the study protocol was reviewed and successfully approved by the Ethics Committee of Sinopharm Dongfeng General Hospital and was waived to informed consent.

\section{Funding}

There is no funding to report.

\section{Disclosure}

The authors declare that they have no competing interests.

\section{References}

1. Ronco C, Bellomo R, Kellum JA. Acute kidney injury. Lancet. 2019;394:1949-1964. doi:10.1016/S0140-6736(19)32563-2

2. Collister D, Pannu N, Ye F, et al. Health care costs associated with AKI. Clin J Am Soc Nephrol. 2017;12:1733-1743. doi:10.2215/CJN.00950117

3. Hu Y, Cao Q, Wang H, et al. Prognostic nutritional index predicts acute kidney injury and mortality of patients in the coronary care unit. Exp Ther Med. 2021;21:123. doi:10.3892/etm.2020.9555

4. Hu Y, Liu H, Fu S, Wan J, Li X. Red blood cell distribution width is an independent predictor of AKI and mortality in patients in the coronary care unit. Kidney Blood Press Res. 2017;42:1193-1204. doi:10.1159/000485866

5. Kellum JA, Sileanu FE, Bihorac A, Hoste EA, Chawla LS. Recovery after acute kidney injury. Am J Respir Crit Care Med. 2017;195:784-791. doi:10.1164/rccm.201604-0799OC

6. Darmon M, Truche AS, Abdel-Nabey M, Schnell D, Souweine B. Early recognition of persistent acute kidney injury. Semin Nephrol. 2019;39:431-441. doi:10.1016/j.semnephrol.2019.06.003

7. Hoste E, Kellum JA, Selby NM, et al. Global epidemiology and outcomes of acute kidney injury. Nat Rev Nephrol. 2018;14:607-625. doi:10.1038/ s41581-018-0052-0

8. Wang Y, Bellomo R. Cardiac surgery-associated acute kidney injury: risk factors, pathophysiology and treatment. Nat Rev Nephrol. 2017;13:697-711. doi:10.1038/nrneph.2017.119

9. Kang JS, Moon KH, Youn YH, Park JS, Ko SH, Jeon YS. Factors associated with postoperative acute kidney injury after hip fractures in elderly patients. J Orthop Surg (Hong Kong). 2020;28:615556525. doi:10.1177/2309499019896237

10. Cho W, Hwang TY, Choi YK, et al. Diastolic dysfunction and acute kidney injury in elderly patients with femoral neck fracture. Kidney Res Clin Pract. 2019;38:33-41. doi:10.23876/j.krcp.18.0083

11. Agar A, Gulabi D, Sahin A, et al. Acute kidney injury after hip fracture surgery in patients over 80 years of age. Arch Orthop Trauma Surg. 2021. doi:10.1007/s00402-021-03969-y

12. Zhou X, Zhang Y, Teng Y, et al. Predictors of postoperative acute kidney injury in patients undergoing hip fracture surgery: a systematic review and meta-analysis. Injury. 2021;52:330-338. doi:10.1016/j.injury.2020.09.060

13. Ganta A, Parola R, Perskin CR, Konda SR, Egol KA. Risk factors and associated outcomes of acute kidney injury in hip fracture patients. $J$ Orthop. 2021;26:115-118. doi:10.1016/j.jor.2021.07.019

14. Li S, Zhang J, Zheng H, Wang X, Liu Z, Sun T. Prognostic role of serum albumin, total lymphocyte count, and mini nutritional assessment on outcomes after geriatric hip fracture surgery: a meta-analysis and systematic review. J Arthroplasty. 2019;34:1287-1296. doi:10.1016/j. arth.2019.02.003

15. Wang X, Dai L, Zhang Y, Lv Y. Gender and low albumin and oxygen levels are risk factors for perioperative pneumonia in geriatric hip fracture patients. Clin Interv Aging. 2020;15:419-424. doi:10.2147/CIA.S241592

16. Bohl DD, Shen MR, Hannon CP, Fillingham YA, Darrith B, Della VC. Serum albumin predicts survival and postoperative course following surgery for geriatric hip fracture. J Bone Joint Surg Am. 2017;99:2110-2118. doi:10.2106/JBJS.16.01620 
17. Zhu X, Cheang I, Liao S, et al. Blood urea nitrogen to creatinine ratio and long-term mortality in patients with acute heart failure: a prospective cohort study and meta-analysis. Cardiorenal Med. 2020;10:415-428. doi:10.1159/000509834

18. Wernly B, Lichtenauer M, Vellinga N, et al. Blood urea nitrogen (BUN) independently predicts mortality in critically ill patients admitted to ICU: a multicenter study. Clin Hemorheol Microcirc. 2018;69:123-131. doi:10.3233/CH-189111

19. Zou XL, Feng DY, Wu WB, Yang HL, Zhang TT. Blood urea nitrogen to serum albumin ratio independently predicts 30-day mortality and severity in patients with Escherichia coli bacteraemia. Med Clin (Barc). 2021;157:219-225. doi:10.1016/j.medcli.2020.06.060

20. Dundar ZD, Kucukceran K, Ayranci MK. Blood urea nitrogen to albumin ratio is a predictor of in-hospital mortality in older emergency department patients. Am J Emerg Med. 2021;46:349-354. doi:10.1016/j.ajem.2020.10.008

21. Tao L, Zhou S, Chang P, An S. Effects of ondansetron use on outcomes of acute kidney injury in critically ill patients: an analysis based on the MIMIC-IV database. J Crit Care. 2021;66:117-122. doi:10.1016/j.jcrc.2021.07.015

22. Kellum JA, Lameire N. Diagnosis, evaluation, and management of acute kidney injury: a KDIGO summary (Part 1). Crit Care. $2013 ; 17: 204$. doi:10.1186/cc11454

23. McKeag P, Spence A, Hanratty B. Acute kidney injury following surgery for hip fracture. Acta Ortop Bras. 2020;28:128-130. doi:10.1590/1413785220202803226779

24. Brauner CJ, Aasbrenn M, Sandoval CL, et al. Predictors of acute kidney injury after hip fracture in older adults. Geriatr Orthop Surg Rehabil. 2020;11:1466796008.

25. Kupeli I, Unver S. The Correlation between preoperative and postoperative hypoalbuminaemia and the development of acute kidney injury with respect to the KDIGO criteria in the hip fracture surgery in elderly patients. Turk J Anaesthesiol Reanim. 2020;48:38-43. doi:10.5152/ TJAR.2019.65642

26. Shin KH, Han SB. Early postoperative hypoalbuminemia is a risk factor for postoperative acute kidney injury following hip fracture surgery. Injury. 2018;49:1572-1576. doi:10.1016/j.injury.2018.05.001

27. Rantalaiho I, Gunn J, Kukkonen J, Kaipia A. Acute kidney injury following hip fracture. Injury. 2019;50:2268-2271. doi:10.1016/j. injury.2019.10.008

28. Li ZC, Pu YC, Wang J, Wang HL, Zhang YL. The prevalence and risk factors of acute kidney injury in patients undergoing hip fracture surgery: a meta-analysis. Bioengineered. 2021;12:1976-1985. doi:10.1080/21655979.2021.1926200

29. Dossetor JB. Creatininemia versus uremia. The relative significance of blood urea nitrogen and serum creatinine concentrations in azotemia. Ann Intern Med. 1966;65:1287-1299. doi:10.7326/0003-4819-65-6-1287

30. Mansour SG, Martin TG, Obeid W, et al. The role of volume regulation and thermoregulation in AKI during Marathon running. Clin J Am Soc Nephrol. 2019;14:1297-1305. doi:10.2215/CJN.01400219

31. Deep A, Saxena R, Jose B. Acute kidney injury in children with chronic liver disease. Pediatr Nephrol. 2019;34:45-59. doi:10.1007/s00467-0183893-7

32. Yu J, Park JY, Ha S, Hwang JH, Kim YK. C-reactive Protein/albumin ratio and acute kidney injury after radical cystectomy among elderly patients: a propensity score-matched analysis. Dis Markers. 2020;2020:8818445. doi:10.1155/2020/8818445

33. Xu L, Li C, Zhao L, et al. Acute kidney injury after nephrectomy: a new nomogram to predict postoperative renal function. BMC Nephrol. 2020;21:181. doi:10.1186/s12882-020-01839-0

International Journal of General Medicine

Dovepress

\section{Publish your work in this journal}

The International Journal of General Medicine is an international, peer-reviewed open-access journal that focuses on general and internal medicine, pathogenesis, epidemiology, diagnosis, monitoring and treatment protocols. The journal is characterized by the rapid reporting of reviews, original research and clinical studies across all disease areas. The manuscript management system is completely online and includes a very quick and fair peer-review system, which is all easy to use. Visit http://www.dovepress.com/testimonials.php to read real quotes from published authors.

Submit your manuscript here: https://www.dovepress.com/international-journal-of-general-medicine-journal 